\title{
LOSS OF CONTRAST SENSITIVITY IN MULTIPLE SCLEROSIS PATIENTS
}

\author{
Deleva N., A. Tzoukeva, I. Dimitrov, A. Kaprelyan \\ Department of neurology, Medical University, Varna, Bulgaria \\ Reviewed by: Prof. D. Minchev, MD, PhD
}

\begin{abstract}
Neuro-ophthalmic impairments are common in multiple sclerosis (MS). Previous reports have shown that contrast sensitivity (CS) may be the most sensitive measure of afferent visual disfunction in patients with MS, even while sparing visual acuity. OBJECTIVE: To investigate CS testing as a method for detection of visual disturbances in MS patients with or without clinical data of retrobulbar neuritis. MATERIALS AND METHODS: CS was measured in 24 patients divided into two groups as follows: 12 cases with clinical data of retrobulbar neuritis $(\mathrm{ON}+)$ and 12 without medical history of retrobulbar neuritis (ON-). RESULTS AND CONCLUSION: CS was abnormal in $91,7 \%$ of the patients from the first group and in $83,3 \%$ of the patients from the second group. Visuograms of mean CS's as a function of the spatial frequency in 30 normal subjects, $12 \mathrm{MS} \mathrm{ON}+$ patients and $12 \mathrm{MS}$ ON-patients. No statistically significant variations were established between the both investigated groups of MS patients. Our study confirms the previously reported abnormalities in CS curve in patients with normal and reduced visual acuity. This report proves the possibility of this test to benefit the earlier diagnosis of Multiple Sclerosis and to improve the detection of latent visual deficit.
\end{abstract}

Keywords: Multiple sclerosis, visual disturbances, contrast sensitivity

Neuro-ophthalmic impairments are common in multiple sclerosis (MS). Optic neuritis ( $\mathrm{ON})$ is a frequent visual sensory disturbance, presenting acute, unilateral and painful visual loss $(2,10,17)$. Careful examination of MS patients with normal visual acuity and no prior history of $\mathrm{ON}$, may also reveal subclinical dysfunction of vision $(1,6,9,11,18)$. This type of complaint such as blurred vision is not detectable with conventional neuro-ophthalmic tests. Evidence from previous reports indicates that contrast sensitivity (CS) may be the most sensitive measures of afferent visual dysfunction in patients with MS, even while sparing visual acuity $(3,4,8,12,13,14,15,16)$.

\section{OBJECTIVE}

To investigate CS testing as a method for detection of visual disturbances in MS patients with or without clinical data of $\mathrm{ON}$.

\section{SUBJECTS AND METHODS}

Thirty normal subjects ( 9 men and 21 women) and 24 patients ( 7 men and 17 women) with clinically definite MS were tested. The mean age of the normal subjects was 37.8 \pm 12.4 , and the mean age of the patients was $36.8 \pm 11.2$. Ac- cording to the clinical criteria the patients were divided into two subgroups: 12 ( 3 men and 9 women with mean age $37.5 \pm 12.1)$ with history of unilateral optic neuritis (ON+) and 12 (4 men and 8 women, mean age $36.1 \pm 10.9)$ without history of $\mathrm{ON}(\mathrm{ON}-)$. All normal subjects and the patients of subgroup $\mathrm{ON}$ - had visual acuity $\geq 1.0$, and all patients of subgroup $\mathrm{ON}+$ had visual acuity $\geq 0.5$.

Patients were examined in standardized procedure and neurological deficit was recorded as a score according to the Expanded Disability Status Scale (EDSS).

CS was measured with vertical sinusoidal grating patterns over the spatial frequency range from 2 to 64 cycles/degree. The objective was to distinguish the target from a homogeneous field. Testing was monocular and the non tested eye was covered with patch. The CS scores obtained for each of the sine wave gratings examined were plotted as a function of target spatial frequency yielding the contrast sensitivity function.

Results: Results of CS testing were compared to those of normal subjects matched for age. The test was abnormal in $91.7 \%$ of patients of subgroup $\mathrm{ON}+$ and in $83.3 \%$ of subgroup ON- (fig. 1)

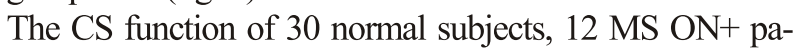
tients and 12 MS ON- patients is depicted in Fig. 2

Globally CS was reduced on the whole spatial frequency range in cases of subgroup $\mathrm{ON}+$ and had selective fre- 
quency losses on the medium and low frequencies in subgroup ON-. No significant CS difference was established between subgroup $\mathrm{ON}+$ and $\mathrm{ON}-$.

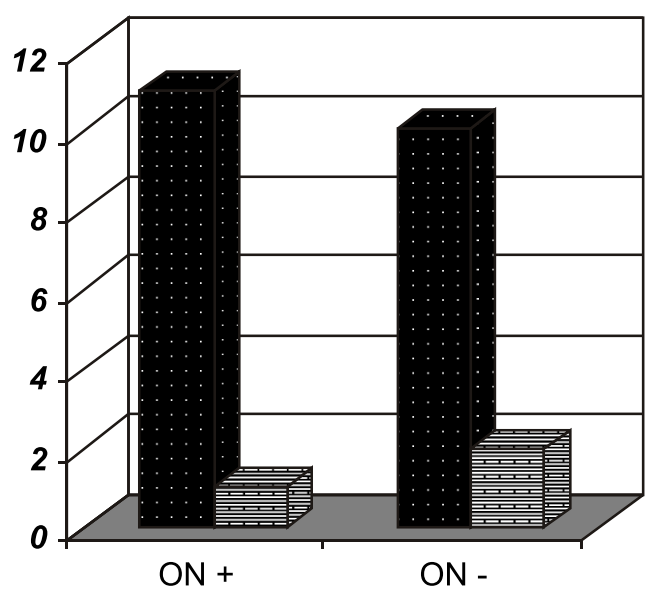

口Abnormal CS 믐 Normal CS

Fig. 1

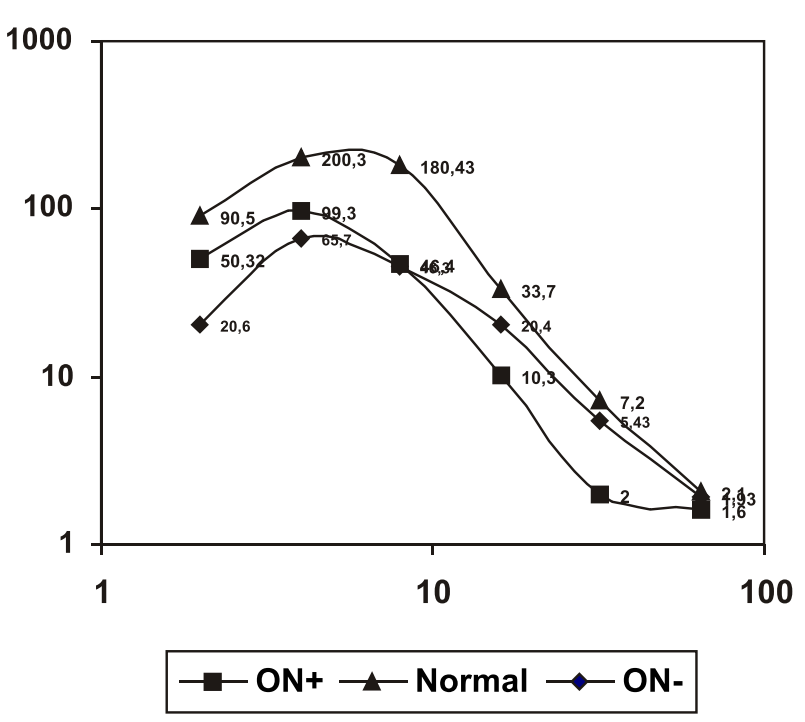

Fig. 2

\section{DISCUSSION}

The spatial resolution of the visual system is usually accessed using a sample measure of visual acuity. Snellen acuity may be a sufficiently sensitive measure of visual change in patients with ON and MS, but may not capture such visual loss in asymptomatic visual dysfunctions, that are quite common in patients with MS $(3,4,6,9,13)$. Numerous studies have demonstrated that CS testing was a highly reliable method of visual assessment in patients wit MS $(4,16,18)$. The results of our study confirm the previous reported abnormalities in CS in patients with normal and re- duced visual acuity. This psychophysical method can measure some aspect of visual function, subclinical visual dysfunction in MS, not captured by visual acuity alone. The selective frequency losses in CS support the hypothesis that an important substrate of this subclinical loss could be lesions of the network relying on myelinated lateral connections of the visual cortex, binding monocular columns $(1,5,7,8,14,15)$. This report proves the possibility of CS testing to benefit the earlier diagnosis of MS when visual disturbances are not clinically evident and to improve the detection of latent visual deficit.

\section{REFERENCES}

1. Antal A; Aita JF; Bodis-Wollner I. The paracentral visual field in multiple sclerosis: evidence for a deficit in interneuronal spatial summation? Vision Res, 2001 Jun, Vol. 41(13). pp. 1735-42.

2. Ashworth B, Aspinall PA, Mitchell JD. Visual function in multiple sclerosis. Doc Ophthalmol 1989 Nov;73(3):209-24.

3. Balcer LJ. Clinical outcome measures for research in multiple sclerosis. J Neuroophthalmol 2001, 21(4):296-301.

4. Bodis-Wollner I. Visual acuity and contrast sensitivity in patients with cerebral lesions. Science 1972;178:769-71.

5. Brannan JR, Bodis-Wolner I. Evidence for two systems mediating perceived contrast. Visual Neuroscience 1991, 6, 587-592.

6. Issa NP; Trepel C; Stryker MP. Spatial frequency maps in cat visual cortex. J Neurosci, 2000 Nov 15, Vol. 20(22). pp. 8504-14.

7. Kupersmith MJ, Nelson JI, Seiple WH, et al. The 20/20 eye in multiple sclerosis. Neurology 1983;33:1015-20.

8. Logi F; Pellegrinetti A; Bonfiglio L; Baglini O; Siciliano G; Ludice A; Sartucci F. Effects of grating spatial orientation on visual evoked potentials and contrast sensitivity in multiple sclerosis. Acta Neurol Scand, 2001 Feb, Vol. 103(2). pp. 97-104.

9. Lycke J, Tollesson PO, Frisen L. Asymptomatic visual loss in multiple sclerosis. J Neurol 2001 Dec;248(12):1079-86.

10. Newman NJ. Multiple sclerosis and related demyelinating diseases. In: Miller NR, Newman NJ, eds. Walsh and Hoyt's Clinical Neuro-Ophthalmology, 5th ed. Baltimore: Williams \& Wilkins, 1998:5539-5676.

11. Regan D, Bartol S, Murray TJ, Beverley KI. Spatial frequency discrimination in normal vision and in patients with multiple sclerosis. Brain $1982 \mathrm{Dec} ; 105$ (Pt 4):735-54.

12. Regan D, Silver R, Murray TJ. Visual acuity and contrast sensitivity in multiple sclerosis--hidden visual loss: an auxiliary diagnostic test. Brain 1977 Sep;100(3):563-79.

13. Regan MP, Regan D. Orientation characteristics of a mechanism in the human visual system sensitive to cyclopean form. Vision Res 2002 Mar;42(5):661-8.

14. Romanova EV, Karlova IZ, Shapiro AL, Shamshinova AM. Psychophysical symptoms of 
demyelinating diseases of the optic nerve in multiple sclerosis. Vestn Oftalmol 2001

Nov-Dec;117(6):29-32.

15. Singh KD; Smith AT; Greenlee MW. Spatiotemporal frequency and direction sensitivities of human visual areas measured using fMRI. Neuroimage, 2000 Nov, Vol. 12(5). pp. 550-64.
16. Trobe JD, Beck RW, Moke PS, et al. Contrast sensitivity and other vision tests in the Optic Neuritis Treatment Trial. Am J Ophthalmol 1996;121:547-53.

17. Vighetto A, Krolak-Salmon P. Inflammatory optic neuropathies. Rev Prat 2001, 15; 51(20):2206-2209.

18. Warner J, Lessell S. Neuro-ophthalmology of multiple sclerosis. Clin Neurosci 1994;2(3-4):180-8. 